\title{
Allopurinol-Induced Drug Reaction with Eosinophilia and Systemic Symptoms Syndrome: A Cause of Acalculous Cholecystitis?
}

\author{
Husnain Waseem ${ }^{1}$, Faisal Inayat ${ }^{2}$, Madina Abduraimova ${ }^{3}$, Stephan Kamholz ${ }^{4}$ \\ 1. Internal Medicine, Maimonides Medical Center, New York, USA 2. Internal Medicine, Allama Iqbal \\ Medical College, Lahore, PAK 3. Internal Medicine, Maimonides Medical Center 4. Pulmonology, \\ Maimonides Medical Center, Brooklyn, USA
}

$\square$ Corresponding author: Husnain Waseem, husnainwaseemkemu@gmail.com Disclosures can be found in Additional Information at the end of the article

\section{Abstract}

Acalculous cholecystitis (AC) is an inflammation of the gallbladder in the absence of gallstones. There are many risk factors associated with AC. However, this report implicates allopurinol as an inciting agent for a severe systemic drug reaction, i.e., the drug reaction with eosinophilia and systemic symptoms (DRESS) syndrome complicated by AC. We report a Chinese woman who presented on two occasions with a diffuse maculopapular rash, elevated liver enzymes, and upper abdominal pain attributable to acute AC, the second episode of which developed after the reintroduction of allopurinol treatment for gout. The AC complicated the DRESS syndrome during the course of her hospitalization.

Categories: Allergy/Immunology, Gastroenterology, Rheumatology

Keywords: dress syndrome, allopurinol, hypersensitivity, acalculous cholecystitis, new entity

\section{Introduction}

Acute acalculous cholecystitis (AC) is defined as a severe gallbladder inflammation without gall-stone-related cystic duct obstruction. It causes $2 \%-15 \%$ of all episodes of acute cholecystitis [1]. The usual predisposing factors associated with the development of AC include recent surgery, burns, trauma, medical illnesses, such as diabetes mellitus, vasculitides such as systemic lupus erythematosus (SLE) or polyarteritis nodosa, congestive heart failure, and cystic artery occlusion [2].

Received 08/02/2017

Review began 08/10/2017 Review ended 08/12/2017 Published 08/16/2017

C) Copyright 2017

Waseem et al. This is an open access article distributed under the terms of the Creative Commons Attribution License CC-BY 3.0., which permits unrestricted use, distribution, and reproduction in any medium, provided the original author and source are credited.

\section{Case Presentation}

A 71-year-old Chinese woman presented to the emergency department (ED) with a generalized body rash of one-day duration. The rash started one day after the patient reinitiated allopurinol treatment for the spontaneous onset of bilateral ankle pain associated with redness and swelling. The patient reported two days of intermittent fever ranging from $100.4{ }^{\circ} \mathrm{F}$ to $101.4^{\circ} \mathrm{F}$. She denied the history of recent travel, tick bite, pain in other joints, and myalgias. Past medical history included hypertension, dyslipidemia, diabetes mellitus, and recently diagnosed gout. She was recently diagnosed with gout and allopurinol therapy was initially started four weeks prior to her current presentation.

The patient revealed that she was hospitalized with a similar rash, high-grade fever, and upper 
abdominal pain two weeks previously. The patient took allopurinol for one week and stopped it after her ankle pain resolved. Physical examination revealed right upper quadrant tenderness, and palpation elicited Murphy's sign (associated with acute cholecystitis). Skin examination revealed a petechial, non-blanching rash on all extremities. A poorly defined, diffuse, and nonscaling erythematous maculopapular rash covered the trunk. The vital signs were-heart rate: 135 beats/min, respiratory rate: $25 / \mathrm{min}$, and temperature: $102.9^{\circ} \mathrm{F}$. In the ED, she developed hypotension of $90 / 60 \mathrm{mmHg}$.

A computed tomography (CT) scan of the abdomen demonstrated nonspecific pericholecystic fluid and periportal edema. Abdominal ultrasonography revealed a thickened gallbladder wall measuring $7 \mathrm{~mm}$ and the presence of pericholecystic fluid. There were no visible stones. No intra or extrahepatic bile duct dilatation was noted. The proximal common bile duct (CBD) measured $3.5 \mathrm{~mm}$. The initial laboratory evaluation revealed white cell count (WBC): $2.3 \mathrm{x}$ $10^{3} / \mu \mathrm{L}\left(4-11 \times 10^{3} / \mu \mathrm{L}\right)$, platelet count: $158 \times 10^{3} / \mu \mathrm{L}\left(150-450 \times 10^{3} / \mu \mathrm{L}\right)$, lactate: $3.9 \mathrm{mEq} / \mathrm{L}(0.5$ $1.3 \mathrm{mEq} / \mathrm{L})$, blood urea nitrogen (BUN): $12 \mathrm{mg} / \mathrm{dL}(10-26 \mathrm{mg} / \mathrm{dl})$, creatinine: $1.4 \mathrm{mg} / \mathrm{dL}(0.6-1.3$ $\mathrm{mg} / \mathrm{dL}$ ), total bilirubin: $2.2 \mathrm{mg} / \mathrm{dL}(0.2-1 \mathrm{mg} / \mathrm{dl})$, direct bilirubin: $0.7 \mathrm{mg} / \mathrm{dL}$ (0 to $0.3 \mathrm{mg} / \mathrm{dL}$ ), aspartate aminotransferase (AST): $71 \mathrm{IU} / \mathrm{L}$ (7-40 IU/L), alanine aminotransferase (ALT): 56 IU/L (0-40 IU/L), alkaline phosphatase (ALP): 100 IU/L (70-230 IU/L), and serum lipase: 45 IU/L (0-140 IU/L). Therein, the patient was diagnosed with acute AC.

Intravenous (IV) vancomycin (1500 mg), cefepime (2 gm), and methylprednisolone (60 mg) were administered in addition to aggressive fluid resuscitation. Percutaneous cholecystostomy and drain placement were accomplished without complications. Blood and urine cultures were persistently negative. Resolution of the rash occurred on Day 3 of hospitalization and, subsequently, the corticosteroid therapy was discontinued. On the fourth hospital day, laboratory data included WBC: $12.9 \times 10^{3} / \mathrm{uL}$, platelets: $52 \times 10^{3} / \mathrm{uL}$, total bilirubin: $2.4 \mathrm{mg} / \mathrm{dL}$, direct bilirubin: $1.5 \mathrm{mg} / \mathrm{dL}$, lactate: $1.3 \mathrm{mEq} / \mathrm{L}$, and creatinine: $0.8 \mathrm{mg} / \mathrm{dL}$. On the fourth, fifth, and sixth hospital days, eosinophil percentages were $17 \%, 14.2 \%$, and $9.5 \%$, respectively. The European Registry of Severe Cutaneous Adverse Reactions (RegiSCAR) score was four, suggesting the possibility of the drug reaction with eosinophilia and systemic symptoms (DRESS) syndrome. Antimicrobial therapy was changed to IV ceftriaxone and IV metronidazole on the fourth day. The patient showed significant improvement in the rash and started tolerating oral feeding. She was discharged from the hospital on Day 8. At the time of discharge, the etiology of acute AC had not been determined with certainty. Allopurinol was not discontinued at the time of discharge.

Five days after hospital discharge, the patient developed left-sided ankle pain and again took one dose of $300 \mathrm{mg}$ allopurinol. Within 24 hours, a diffuse erythematous rash all over her body recurred, and she returned to the emergency department. Examination revealed temperature: $103.7^{\circ} \mathrm{F}$, heart rate: 117 beats/minute, respiratory rate: 20/minute, and blood pressure: 161/61 mmHg. A morbilliform rash was present over the pubic symphysis. A petechial rash was noted on both legs (Figure 1) as well as on the upper extremities (Figure 2). 


\section{Cureus}

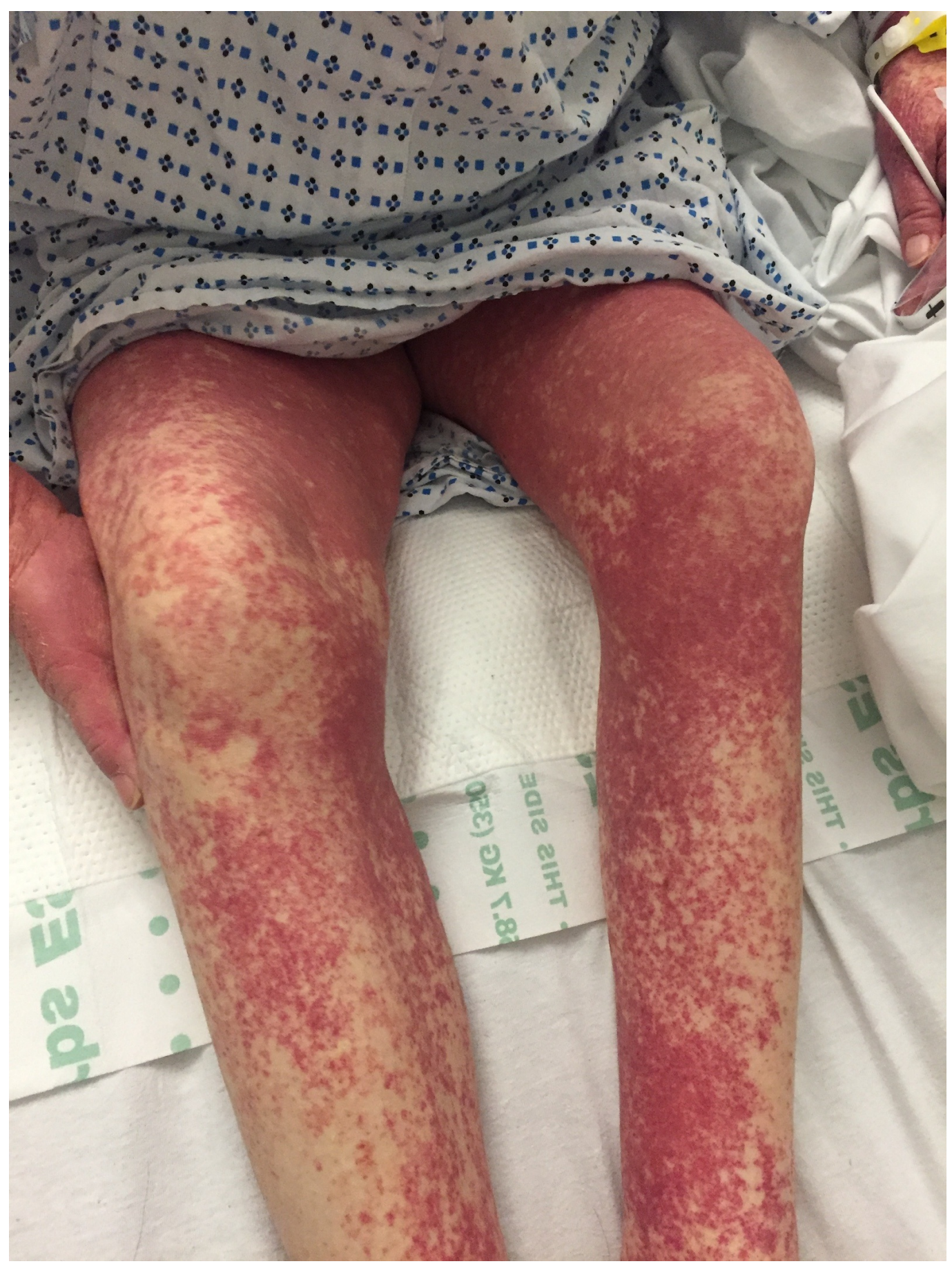

FIGURE 1: Rash on lower extremities at the time of presentation 


\section{Cureus}

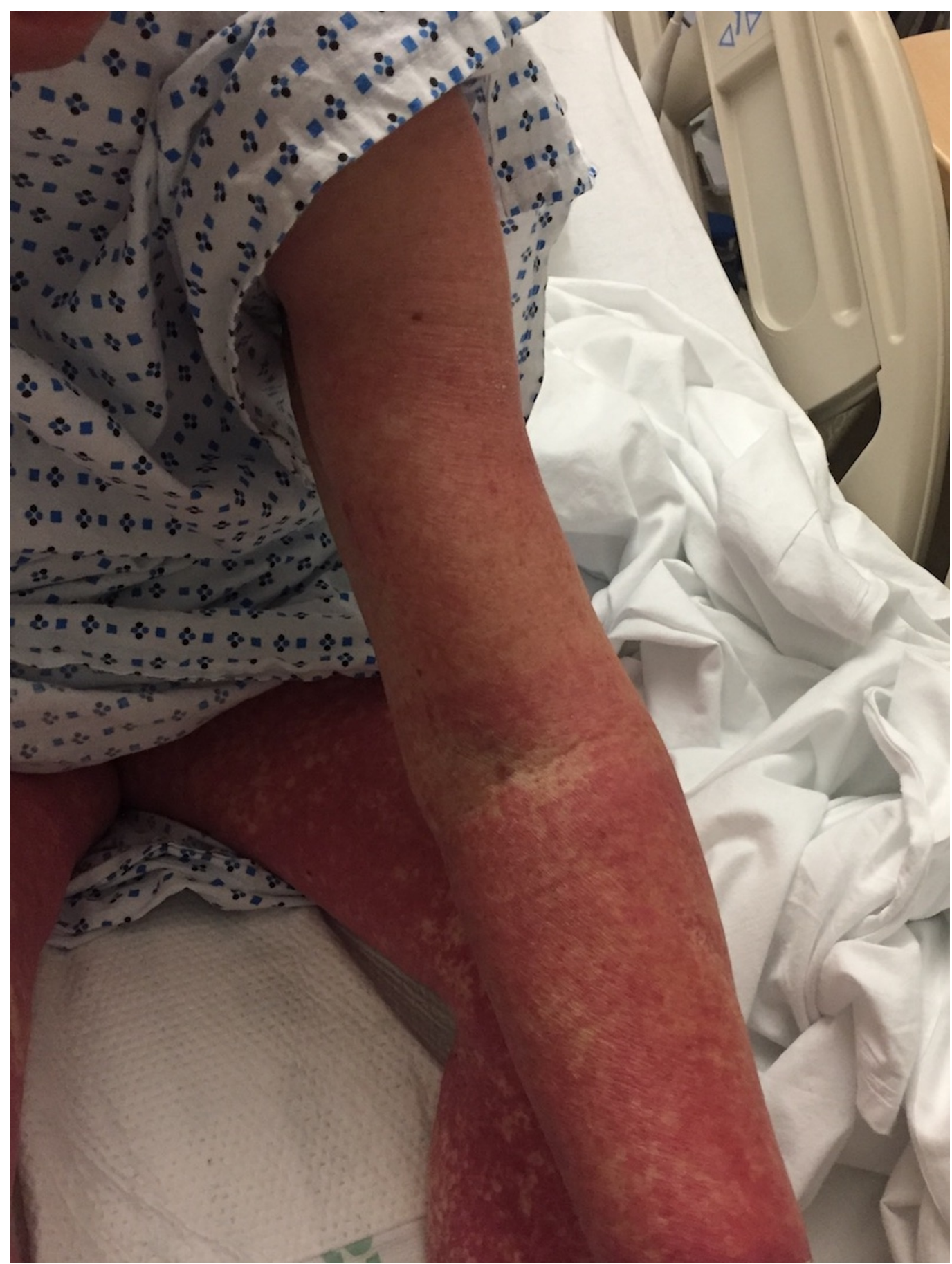

FIGURE 2: Rash on upper extremity at the time of admission

The oral mucosa was uninvolved. The left ankle was warm and tender to touch and a mild effusion was present. Laboratory data included WBC: $9.4 \times 103 / \mathrm{LL}(4-11 \times 103 / \mu \mathrm{L})$, eosinophil: 31\% (1\%-3\%), neutrophils: 72.5\% (57-67\%), C-reactive protein: (CRP) $9.100 \mathrm{mg} / \mathrm{dL}$ (1-3 mg/dL), erythrocyte sedimentation rate (ESR): $90 \mathrm{~mm} / \mathrm{hr}(0-20 \mathrm{~mm} / \mathrm{hr})$, lipase: $170 \mathrm{U} / \mathrm{L}(0-140 \mathrm{U} / \mathrm{L})$, Btype natriuretic peptide (BNP): $177 \mathrm{pg} / \mathrm{mL}(0-100 \mathrm{pg} / \mathrm{mL})$, and uric acid: $5.9 \mathrm{mg} / \mathrm{dL}$ (2.1-6.3 $\mathrm{mg} / \mathrm{dl})$. Radiographic imaging of the chest, abdomen, and pelvis did not reveal any abnormalities. The diagnosis of "possible" DRESS syndrome due to allopurinol hypersensitivity was made on a RegiSCAR score of three. Intravenous methylprednisolone (40 mg) was administered twice daily for six to seven days. Significant resolution of the rash occurred during treatment. The upper left arm was almost cleared of the rash (Figure 3). Residual post- 


\section{Cureus}

inflammatory changes were noted on both legs (Figure 4). Corticosteroid therapy was discontinued. The patient was educated about her allergy to allopurinol and about not taking this medication in the future.

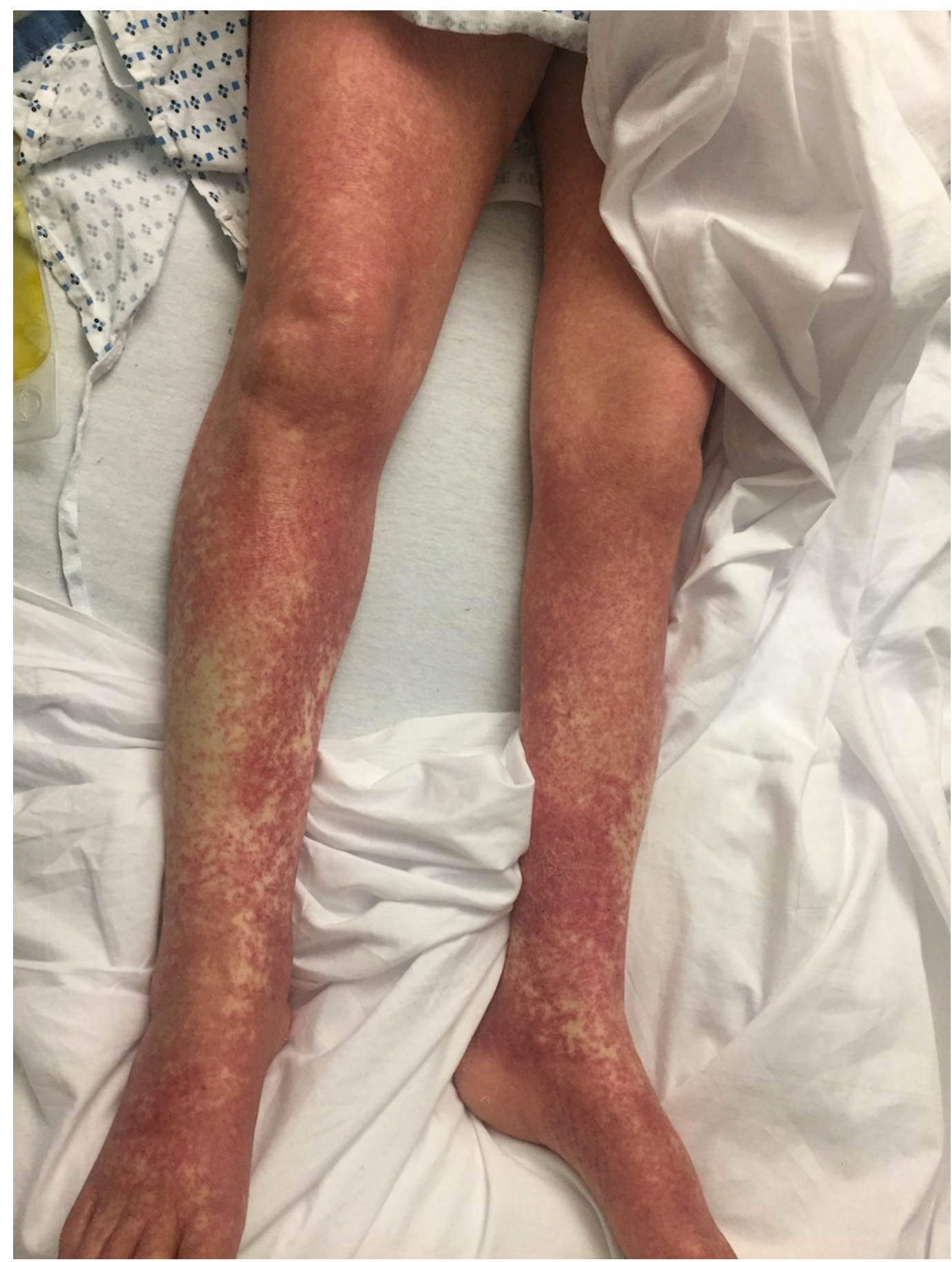

FIGURE 3: Resolving erythematous lesions on lower limbs at the time of discharge 


\section{Cureus}

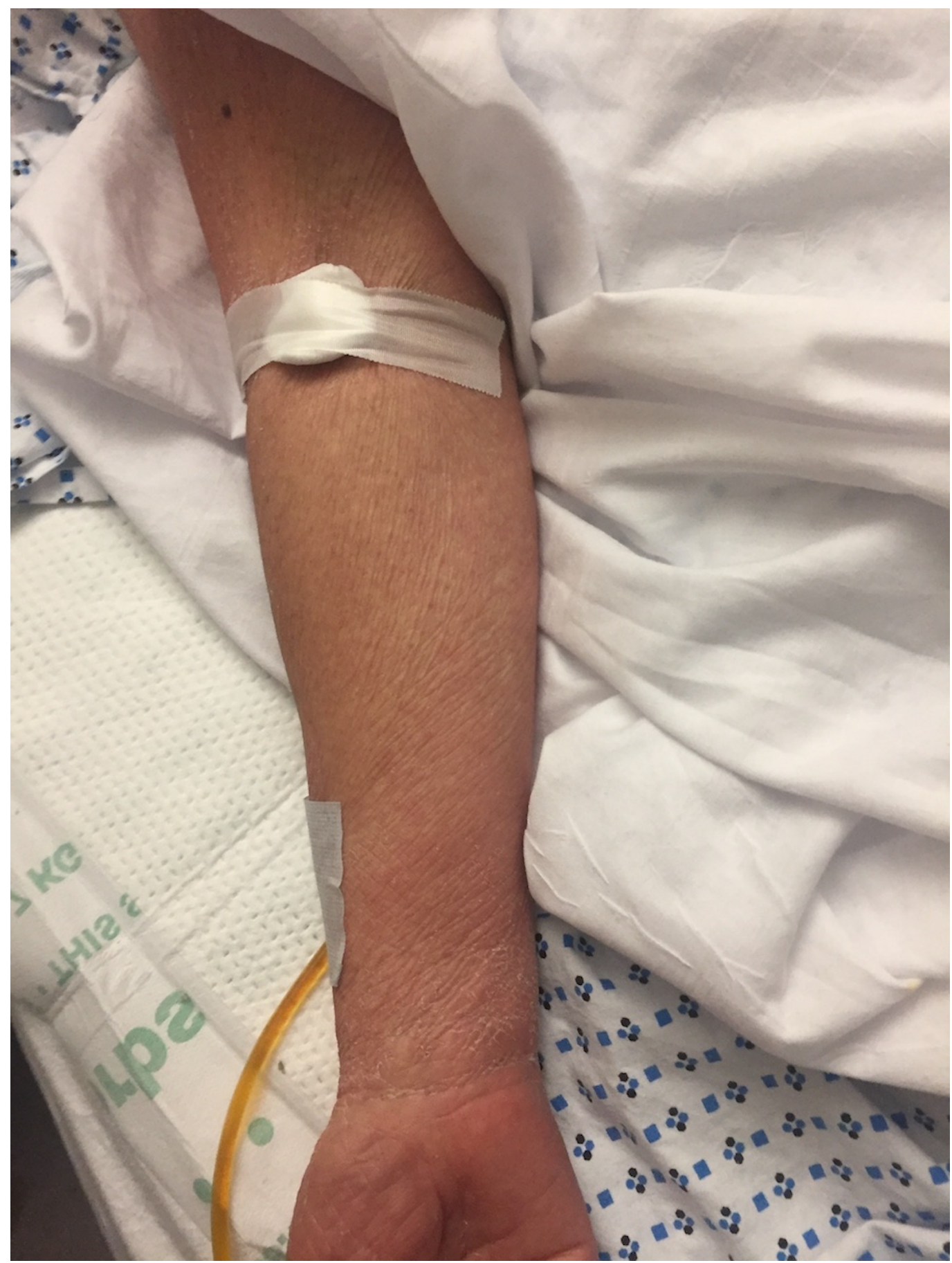

FIGURE 4: Resolution of rash on upper limb at discharge

\section{Discussion}

Allopurinol hypersensitivity is a rare adverse reaction that is characterized by cutaneous manifestations, ranging from benign maculopapular eruptions to serious disorders such as Stevens-Johnson syndrome and toxic epidermal necrolysis. The reaction may be accompanied by fever, hepatic dysfunction, renal impairment, eosinophilia, and leukocytosis [3]. One of the clearest risk factors for developing allopurinol toxicity is Han-Chinese ethnicity, especially those individuals carrying the human leukocyte antigen (HLA)-B 5801 alleles [3-5]. Although HLA phenotyping was not available for this patient, she was of Han-Chinese descent. 
The DRESS syndrome was first described in 1996. Fever, lymphadenopathy, rash, and internal organ involvement with marked eosinophilia are common manifestations of this disease. The most frequently involved organ is the liver, followed by the kidney and lungs. Allopurinol was found to be the culprit drug in $19 \%$ of the cases in a literature review of 172 cases [6]. The pathophysiology of the DRESS syndrome remains unclear, but a defect in the detoxification of the causative drug, immunological imbalance, and infections such as human herpes virus type 6 (HHV 6) are previously suggested [7]. The overall mortality in DRESS is about $10 \%$ and occurs in patients with severe multiorgan involvement.

The absence of gallstones on abdominal imaging and studies performed at the time of initial admission mitigated against acute gallstone-induced cholecystitis. The patient did not have common risk factors for AC such as trauma, burns, recent surgery, and sepsis, as blood and urine cultures were persistently negative. Because the association of the symptoms and signs attributable to allopurinol were not noted during the initial hospitalization, the drug was not discontinued. We hypothesize that the systemic hypersensitivity reaction, systemic inflammatory response syndrome (SIRS), and DRESS syndrome secondary to allopurinol toxicity could have been the cause of this patient's AC.

It is likely that our patient developed AC during the first hospital admission due to a hypersensitivity reaction caused by allopurinol. There are several reasons to support this contention: (a) The patient was recently started on allopurinol four weeks prior to the first episode of rash and right upper quadrant abdominal pain. One report suggested that the median time to the onset of allopurinol hypersensitivity was 30 days [4]. (b) This patient manifested a similar rash on both occasions. (c) No alternative etiology of AC was identified on imaging studies, and the blood cultures were persistently negative. (d) The patient again developed a similar rash one day after she resumed allopurinol because she was not instructed to stop the medication at the time of her discharge.

The pathogenesis of AC involves endothelial injury, gallbladder ischemia, and stasis, leading to a concentration of bile salts, gallbladder distension, and, eventually, necrosis of the gallbladder tissue. Once AC is established, secondary infection with enteric pathogens, including Escherichia coli, Enterococcus faecalis, Klebsiella spp, Pseudomonas spp., Proteus spp., and Bacteroides spp. is common. Gallbladder perforation occurs in severe cases. The presence of a characteristic rash, the recent use of allopurinol, and the co-development of AC allows us to speculate that AC was related to the allopurinol-induced hypersensitivity reaction. Endothelial injury, bladder ischemia, a release of inflammatory cytokines, such as Interleukin (IL)-1, IL-6, and tumor necrosis factor (TNF), and stasis secondary to SIRS and the DRESS syndrome could have caused the insults to the gallbladder.

The treatment of AC includes the initiation of antibiotics after obtaining blood cultures. Cholecystostomy is often preferred over cholecystectomy as a less invasive option in critically ill patients. Delay in treatment can result in gallbladder perforation and gangrene and mortality rates may be as high as $70 \%$ in such patients. While the placement of a cholecystostomy tube is common, cholecystectomy should be performed if there are findings suggesting gallbladder necrosis, emphysematous cholecystitis, or perforation. These findings were absent in our patient.

\section{Conclusions}

The present report highlights the importance of thorough medication history in patients presenting with symptoms of AC, especially in those with no known cause of gallbladder inflammation. A systemic hypersensitivity reaction associated with drug allergy, such as the DRESS syndrome, may cause AC. Therefore, clinicians should maintain a high index of suspicion, especially in patients taking one of the drugs commonly known to cause severe 
systemic hypersensitivity reactions such as allopurinol.

\section{Additional Information \\ Disclosures}

Human subjects: Consent was obtained by all participants in this study. Conflicts of interest: In compliance with the ICMJE uniform disclosure form, all authors declare the following:

Payment/services info: All authors have declared that no financial support was received from any organization for the submitted work. Financial relationships: All authors have declared that they have no financial relationships at present or within the previous three years with any organizations that might have an interest in the submitted work. Other relationships: All authors have declared that there are no other relationships or activities that could appear to have influenced the submitted work.

\section{References}

1. Treinen C, Lomelin D, Krause C, et al.: Acute acalculous cholecystitis in the critically ill: risk factors and surgical strategies. Langenbecks Arch Surg. 2015, 400:421-427. 10.1007/s00423014-1267-6

2. Barie PS, Eachempati SR: Acute acalculous cholecystitis. Gastroenterol Clin North Am. 2010, 39:343-357. 10.1016/j.gtc.2010.02.012

3. Ramasamy SN, Korb-Wells CS, Kannangara DR, et al.: Allopurinol hypersensitivity: a systematic review of all published cases, 1950-2012. Drug Saf. 2013, 36:953-980. 10.1007/s40264-013-0084-0

4. Hiransuthikul A, Rattananupong T, Klaewsongkram J, et al.: Drug-induced hypersensitivity syndrome/drug reaction with eosinophilia and systemic symptoms (DIHS/DRESS): 11 years retrospective study in Thailand. Allergol Int. 2016, 65:432-438. 10.1016/j.alit.2016.04.001

5. Tsai TF, Yeh TY: Allopurinol in dermatology. Am J Clin Dermatol. 2010, 11:225-232. 10.2165/11533190-000000000-00000

6. Cacoub P, Musette P, Descamps V, et al.: The DRESS syndrome: a literature review . Am J Med. 2011, 124:588-597. 10.1016/j.amjmed.2011.01.017

7. Lee JH, Park HK, Heo J, et al.: Drug rash with eosinophilia and systemic symptoms (DRESS) syndrome induced by celecoxib and anti-tuberculosis drugs. J Korean Med Sci. 2008, 23:521525. 10.3346/jkms.2008.23.3.521 